\title{
ENFERMEDADES MUSCULOESQUELÉTICAS EN CUELLO, MANO/MUÑECA Y FACTORES ASOCIADOS EN ESTUDIANTES DE MEDICINA USUARIOS DE TELÉFONOS CELULARES
}

\author{
MUSCULOSKELETAL DISEASES IN THE NECK, HAND / WRIST AND ASSOCIATED \\ FACTORS, AMONG MEDICAL STUDENTS, CELL PHONE USERS
}

\author{
Frander Oneyde Gutiérrez-Sandres* \\ Jairo Leonardo Palma-Escoto** \\ Indiana López-Bonilla ${ }^{* * *}$ \\ Lilliam López-Narváez ${ }^{* * * *}$
}

\begin{abstract}
Resumen: Los teléfonos móviles son los dispositivos electrónicos portátiles más populares en la actualidad, ofreciendo múltiples usos, tales como entretenimiento, envío de mensajes de textos o para realizar actividades académicas. El uso de estos se ha relacionado con los riesgos disergonómicos. El objetivo de este estudio fue determinar los factores asociados a las enfermedades musculoesqueléticas en cuello, mano/muñeca, en 60 estudiantes de medicina usuarios de teléfonos celulares. Se aplicó una encuesta en línea, junto con un examen físico dirigido a cuello y manos, y filmaciones durante el uso del teléfono móvil. Se encontró una prevalencia del 55\% y 42,4\% de síntomas y enfermedades musculoesqueléticas respectivamente, principalmente, cervicalgia. Se identificó flexión de cuello mayor de $20^{\circ}$ al utilizar el teléfono móvil. Más de la mitad refirió dolor musculoesquelético al usar celulares con pesos mayor o igual a 170 gramos y con espesor de $8 \mathrm{~mm}$; las aplicaciones más usadas fueron whatsapp y facebook. Se encontró, siete veces más probabilidad de cervicalgia por el envío de más de 150 SMS al día, y 1,5 más probabilidad de presentar dolor por el uso mayor o igual a 8 horas. Los hallazgos reflejan que las características del celular, el uso por tiempo prolongado y los riesgos disergonómicos, están relacionados con los síntomas y enfermedades musculoesqueléticas, lo que muestra la importancia de realizar programas de prevención de enfermedades del aparato musculoesquelético y sensibilización a la población y otros usuarios de teléfonos celulares, para contribuir a la reducción de estas enfermedades en una población joven en el futuro.
\end{abstract}

Palabras clave: Factores disergonómicos, teléfonos celulares, cervicalgia, síndrome del túnel del carpo, flexión de cuello, movimientos repetitivos.

*Centro de Investigación en Salud, Trabajo y Ambiente, Facultad de Ciencias Médicas, Universidad Nacional Autónoma de Nicaragua. León, Nicaragua. Correo electrónico: frandergutierrez4@gmail.com. Orcid: https://orcid.org/oooo-0002-0816-6609

${ }^{* *}$ Centro de Investigación en Salud, Trabajo y Ambiente, Facultad de Ciencias Médicas, Universidad Nacional Autónoma de Nicaragua. León, Nicaragua. Correo electrónico: leonardopalmascotto@gmail.com. Orcid: https://orcid.org/oooo-0oo3-1478-6166

${ }^{* * *}$ Centro de Investigación en Salud, Trabajo y Ambiente, Facultad de Ciencias Médicas, Universidad Nacional Autónoma de Nicaragua. León, Nicaragua. Correo electrónico: indianalopezb@gmail.com. Orcid: https://orcid.org/o00o-0002-0760-1111

${ }^{* * * *}$ Centro de Investigación en Salud, Trabajo y Ambiente, Facultad de Ciencias Médicas, Universidad Nacional Autónoma de Nicaragua. León, Nicaragua. Correo electrónico: ae26ld14@gmail.com. Orcid: https://orcid.org/oooo-0003-4986-7644. Autor de correspondencia. 


\begin{abstract}
Cellphones are the portable electronic devices more popular nowadays, with multiple uses such as entertainment, text messages or to carry on academic activities. Its use has been related to dysergonomic risks. The objecticve of this study was to determine the factors linked to musculoskeletal diseases in the neck, hand/wrist, in 60 medicine students, cellphone users. It was applied an online survey, a physical exam focused on neck and hands was made; and filming while using cellphones. It was found prevalence of $55 \%$ and $42.5 \%$ of musculeskeletals symptoms and diseases respectively, mainly Cervicalgia. It was identified neck flexion over $20^{\circ}$ when using cellphone. More than the half referred musculoskeletal pain when using mobile phones with weight over or equal to 170 grams and 8 mm thickness; the most used apps were whatsapp and facebook. It was found, seven times more probability of cervicalgia for sending more than 150 messages daily; and 1.5 more probability of presenting pain for the use over or equal to 8 hours. The findings reflect that the characteristics of the cell phone, long-term use and the dysergonomic risks are related to the symptoms and musculoskeletal diseases, these show us the importance of carrying out programs for the prevention of diseases of the musculoskeletal system and sensitization to the study population and other cell phone users, to contribute to the reduction of these diseases in a young population, in the future.
\end{abstract}

Keywords: Dysergonomic factors, cell phones, cervicalgia, carpal tunnel syndrome, neck flexion, repetitive movements.

Recepción: 08.10.2021 / Revisión: 19.10.2021 / Aceptación: 23.11.2021

\title{
Introducción
}

Actualmente, en la sociedad digital, el uso de teléfonos inteligentes ha aumentado rápidamente, para el segundo trimestre del 2021 se reportó 8,1 mil millones de usuarios de teléfonos inteligentes en todo el mundo (Ericsson Mobility Report, 2021, junio). Este dispositivo tecnológico es usado por la mayoría de la población en general, por sus amplias funciones y diversas comodidades, como enviar y recibir correos eléctronicos, mensajes y acceder a internet para entretenimiento (Perera, 2012). Sin embargo, el uso de teléfonos inteligentes es asociado con síntomas fisicos en algunos usuarios. En un estudio que investigaron a dos grupos, los que utilizaban teléfonos móviles para el ocio, y los que utilizaban computadoras u otros dispositivos, reflejaron que ambos grupos presentaban con mucha frecuencia síntomas del aparato musculoesquelético. Los que usaban los teléfonos móviles presentaban síntomas muy a menudo, principalmente en muñeca, dedos, hombros, cadera y espalda (Korpinen \& Pääkkönen, 2009).

Por otra parte, un estudio realizado en estudiantes de carrera de la salud, mostró en el análisis de los predictores de la gravedad, que la edad y la duración del uso del celular se asociaron significativamente con la gravedad del dolor de cuello, mientras que solamente la duración del uso del teléfono se asoció significativamente con la duración del dolor (AlHadidi, et al., 2019). Así mismo, en un estudio de seguimiento, se encontraron asociaciones entre enviar mensajes de texto y síntomas en cuello y extremidades superiores, en adultos jóvenes entre 20 a 24 años, en Suecia (Gustafsson et al., 2017).

Estudio cuyo objetivo fue evaluar el movimiento, actividad y posturas del dedo pulgar mientras enviaba mensaje con el teléfono celular, mostró que la postura de pie o sentada y el 
tipo de tarea como sostener el teléfono y enviar mensajes afectaron la actividad muscular y la postura del dedo pulgar. También, se demostró mayor actividad en el extensor de los dedos y el abductor largo al ingresar mensaje, y mayor abducción en las mujeres en comparación con los hombres (Gustafsson et al., 2010).

Es importante mencionar que, este tema está bien estudiado a nivel mundial y en diferentes usuarios de teléfonos celulares, sin embargo, en Nicaragua no se ha realizado ninguna investigación al respecto, y en vista que en la actualidad el teléfono celular se ha convertido en una herramienta para el aprendizaje en los estudiantes, los cuales utilizan este dispositivo para realizar búsquedas, para elaborar sus tareas o informes y también para el ocio por tiempo prolongado, exponiéndose de esa manera a riesgos disergonómicos como la adopción de posturas forzadas, estáticas y movimientos repetitivos, entre otros, se consideró un tema de mucha relevancia y de prioridad para investigar. El objetivo principal de esta investigación fue determinar los factores asociados a las enfermedades musculoesqueléticas en cuello, mano/muñeca, en estudiantes usuarios de teléfonos celulares, de la carrera de Medicina, de la Universidad Nacional Autónoma de Nicaragua (UNAN-León), con el fin de proponer medidas de prevención de las enfermedades musculoesqueléticas principalmente en población joven.

\section{Materiales y métodos}

Estudio transversal donde se evaluó la presencia de enfermedades musculoesqueléticas en mano/muñeca y cuello y los factores asociados por el uso del teléfono celular, en 60 estudiantes del sexto año de la carrera de Medicina, de la Facultad de Ciencias Médicas de la UNAN-León, en el año 2020. Se incluyeron a los estudiantes que tenían más de un año de utilizar teléfono celular y se excluyeron a los estudiantes que tenían historia de lesiones traumáticas, intervenciones quirúrgicas o condiciones médicas previamente diagnosticadas en cuello y mano/muñeca.

En vista de que la Universidad realizó un reorganización de las clases de modo presencial a modo virtual debido a la pandemia del COVID-19, la selección de los estudiantes se hizo solamente con los que llegaban de manera presencial a las prácticas clínicas en el hospital y que cumpliesen con los criterios previamente establecidos.

El instrumento utilizado para la recolección de datos fue un cuestionario que consta de tres secciones, los datos generales, características del celular (peso, longitud, ancho, espesor y modelo), uso del celular (tiempo de uso, propósito, tiempo y escritura de mensajes, dominio y manipulación del teléfono y uso de otros dispositivos), la sección de los síntomas y enfermedades musculoesqueléticas y la sección de identificación de los riesgos disergonómicos de cuello y mano/muñeca.

Previo permiso de las autoridades facultativas y de los participantes del estudio, se realizó una prueba piloto con 10 estudiantes, con el fin de verificar el tiempo de duración de recolección de datos por cada estudiante y la claridez y facilidad de aplicación de los instrumentos en las tres etapas del proceso. En la primera etapa, se aplicó utilizando el 
formulario de Google en línea. En la segunda etapa, se realizó al examen físico, solamente a los estudiantes que referían presentar dolor o molestia musculoesquelética en cuello y mano/muñeca.

En cuello se valoró limitación de movimiento, graduación muscular y dolor a la flexión, extensión, inclinación lateral, rotación y palpación de puntos dolorosos. El examen físico de mano/muñeca se exploró a través del test de tinnel, test de flick, test de phalen, de abducción, entre otros. En la tercera etapa, se realizó filmación a cada estudiante durante un minuto aproximadamente mientras utilizaban el teléfono celular; previamente se orientó a los participantes a que utilizaran el celular de la manera que lo hacían habitualmente. La filmación se realizó con el estudiante en posición de pie y en diferentes ángulos; se utilizó una videocámara de acción $4 \mathrm{~K}$ Full HD/3ofps.

Para la identificación de los riesgos disergonómicos, se observaron los videos las veces que fueron necesarias. Para una mejor identificación de los ángulos de las diferentes posturas y movimientos en cuello y mano/muñeca se utilizó el programa Kinovea, que es un reproductor de video para análisis deportivo, que entre sus funciones mide ángulos, distancias y tiempos.

Previo a la recolección de datos, los investigadores fueron entrenados en el examen físico dirigido a cuello y mano/muñeca por un especialista en ortopedia, y para el análisis e identificación de los riesgos disergonómicos, por una especialista en ergonomía.

Se realizó un análisis descriptivo de todas las variables, se calculó frecuencia, porcentaje y promedio a las variables cuantitativas. Para evaluar la posible relación entre los factores asociados a los síntomas y enfermedades musculoesqueléticas, se calculó el chi cuadrado, considerando relación positiva, si el valor de $\mathrm{P}$ es $<0,05$, la razón de prevalencia (RP) y el intervalo de confianza del 95\%.

En relación a los aspectos éticos, se solicitó permiso a través de un consentimiento informado a cada participante, previa explicación de la importancia de la investigcion y cuál sería su participación.

\section{Resultados y discusión}

Este es el primer estudio en Nicaragua que investiga la prevalencia y factores asociados a síntomas y enfermedades musculoesqueléticas en cuello y mano/muñeca, en usuarios de teléfonos celulares. De los 60 estudiantes, el 58,3\% eran hombres y el $41 \%$ mujeres, con edad promedio de 23,18 años, y el 73,3\%, edad entre 21 y 23 años.

En relación a las características del teléfono celular, todos son de pantalla táctil, con peso mayor o igual a 170 gr y longitud mayor o igual a $150 \mathrm{~mm}$ y con uso de más de 8 horas por día. De acuerdo al propósito, fue principalmente para WhatsApp, Facebook y Google. La mayoría, el 78\%, lo utilizan todo el tiempo (tabla 1).

Similares características y uso de los teléfonos celulares fueron investigadas en la Universidad Khon Kaen, Tailandia, y aunque la muestra de esta fue mayor que el presente 
estudio se encontraron similitudes en el ancho, grosor de los celulares, los minutos que duraba mientra lo utilizaba, propósitos de uso y horas al día utilizando el celular. Diferencias mayores se encontraron en el peso, longitud, años de uso y tiempo de descanso (Namwongsa et al., 2018).

Por otro lado, el presente estudio reflejó que el 55\% de los estudiantes presentaron síntomas musculoesqueléticos al usar el teléfono celular por 9 horas o más al día; coincidiendo con los resultados descritos por Oliveira et al. (2016), que los que utilizan de 810 horas el teléfono, tienen mayor relación con la presencia de sintomatología musculoesquelética. Resultados similares presentan Correa-Botero et al. (2017), en estudio realizado en universidades privadas de Medellín, en relación al uso del "smartphone" entre 58 horas, reflejando que tienen mayor tendencia a presentar sintomatología musculoesquelética por encima del $36,6 \%$.

Tabla 1. Características, tiempo de uso y propósito de los teléfonos celulares.

\begin{tabular}{|c|c|c|c|}
\hline Características & $\mathbf{n}$ & $\%$ & $\overline{\mathbf{x}}$ \\
\hline $\begin{array}{l}\text { Peso (gr) } \\
<170 \\
>0=170\end{array}$ & $\begin{array}{l}33 \\
27\end{array}$ & $\begin{array}{l}55 \\
45\end{array}$ & 168,4 \\
\hline $\begin{array}{l}\text { Longitud (mm) } \\
<150 \\
>0=150\end{array}$ & $\begin{array}{r}28 \\
32 \\
\end{array}$ & $\begin{array}{l}46,6 \\
53,3\end{array}$ & 150,4 \\
\hline $\begin{array}{l}\text { Anchura (mm) } \\
<73 \\
>0=73\end{array}$ & $\begin{array}{l}33 \\
27\end{array}$ & $\begin{array}{l}55 \\
45\end{array}$ & 72,8 \\
\hline $\begin{array}{l}\text { Espesor }(\mathbf{m m}) \\
<8 \\
>0=8\end{array}$ & $\begin{array}{l}36 \\
24\end{array}$ & $\begin{array}{l}60 \\
40\end{array}$ & 8,0 \\
\hline $\begin{array}{l}\text { Tiempo de uso } \\
\text { Años de uso } \\
<8 \\
>0=8\end{array}$ & $\begin{array}{l}33 \\
27\end{array}$ & $\begin{array}{l}55 \\
45\end{array}$ & 8,2 \\
\hline $\begin{array}{l}\text { Minutos utilizando el teléfono al día } \\
<9 \\
>0=9\end{array}$ & $\begin{array}{l}34 \\
26\end{array}$ & $\begin{array}{l}56,7 \\
43,3\end{array}$ & 8,8 \\
\hline $\begin{array}{l}\text { Descanso } \\
\text { Sí }\end{array}$ & 46 & 76,7 & \\
\hline $\begin{array}{l}\text { Tiempo de descanso (minutos) } \\
<15 \text { minutos } \\
>0=15 \text { minutos }\end{array}$ & $\begin{array}{c}4 \\
42\end{array}$ & $\begin{array}{l}67 \\
70\end{array}$ & 95,2 \\
\hline
\end{tabular}




\begin{tabular}{|l|c|c|c|}
\hline Momento que utiliza el teléfono & 6 & 10,0 & \\
Mañana & 8 & 13,3 & \\
Tarde & 11 & 18,3 & \\
Noche & 47 & 78,3 & \\
Todo el tiempo & 58 & 96,7 & \\
\hline Propósitos & 46 & 76,7 & \\
WhatsApp & 43 & 71,7 & \\
Facebook & 40 & 66,7 & \\
Google & 38 & 63,3 & \\
Lectura & 38 & 63,3 & \\
Youtube & 34 & 56,7 & \\
Otros propósitos (juegos y tareas) & & & \\
Instagram & 40 & 66,7 & \\
\hline Horas por día utilizando el celular & 20 & 33,3 & \\
> o = 8 horas & & \\
< 8 horas & &
\end{tabular}

Nota. $\mathrm{n}=60$. Cuestionario en línea.

Tabla 2. Dominio y manipulación del teléfono celular.

\begin{tabular}{|l|c|c|}
\hline Manipulación del teléfono celular & n & \% \\
\hline Mano derecha & 30 & 50 \\
Mano izquierda & 2 & 3,3 \\
Ambas manos & 28 & 46,7 \\
\hline Pulgar derecho & 26 & 43,3 \\
Pulgar izquierdo & 3 & 5,0 \\
Ambos pulgares & 32 & 53,3 \\
\hline Índice derecho & 4 & 6,7 \\
Índice izquierdo & 1 & 1,7 \\
\hline Altura que usa el teléfono celular & \multicolumn{2}{|c|}{} \\
\hline Arriba del pecho o tórax & 15 & 25 \\
\hline A nivel del pecho o tórax & 35 & 58,3 \\
\hline Debajo del pecho o tórax & 16 & 26,7 \\
\hline Postura preferente al usar el teléfono celular & \multicolumn{2}{|c|}{} \\
\hline Sentado & 16 & 76,7 \\
\hline De pie & 14 & 23,3 \\
\hline Caminando & 51 & 85 \\
\hline Acostado & & \\
\hline
\end{tabular}

Nota. Cuestionario en línea.

Según dominio, la mitad de los participantes utilizan la mano derecha y ambos pulgares. El 58,3\% colocan a nivel del pecho o tórax el celular cuando lo utilizan. Y la mayoría, el $76,7 \%$ y $85 \%$, de preferencia lo utilizan en posición sentado y acostado respectivamente. 
Tabla 3. Porcentaje de las características y tiempo del envío de mensajes.

\begin{tabular}{|c|c|c|c|}
\hline Envío de mensajes & $\mathbf{n}$ & $\%$ & $\bar{x}$ \\
\hline $\begin{array}{l}\text { Número de mensajes de texto en un día } \\
>0=150 \text { mensajes } \\
<150 \text { mensajes }\end{array}$ & $\begin{array}{l}31 \\
29\end{array}$ & $\begin{array}{l}51,7 \\
48,3\end{array}$ & 74,7 \\
\hline $\begin{array}{l}\text { Número de mensajes en una hora } \\
>0=50 \text { mensajes } \\
<50 \text { mensajes }\end{array}$ & $\begin{array}{l}42 \\
18\end{array}$ & $\begin{array}{l}70 \\
30\end{array}$ & 62,0 \\
\hline $\begin{array}{l}\text { Número de mensajes de texto enviados en un } \\
\text { minuto } \\
>0=5 \text { mensajes } \\
<5 \text { mensajes }\end{array}$ & $\begin{array}{l}46 \\
14\end{array}$ & $\begin{array}{l}76,7 \\
23,3\end{array}$ & 4,85 \\
\hline $\begin{array}{l}\text { Palabras escritas en un minuto } \\
>0=30 \text { palabras } \\
<30 \text { palabras }\end{array}$ & $\begin{array}{l}33 \\
27\end{array}$ & $\begin{array}{l}55 \\
45\end{array}$ & 39,83 \\
\hline $\begin{array}{l}\text { Tiempo en segundos que tarda escribiendo los } \\
\text { mensajes } \\
>0=30 \mathrm{seg} \\
<30 \mathrm{seg}\end{array}$ & $\begin{array}{l}33 \\
27\end{array}$ & $\begin{array}{l}55 \\
45\end{array}$ & 57,0 \\
\hline
\end{tabular}

Los datos presentados en la tabla 3, reflejan que más de la mitad de los estudiantes, envían más de 150 mensajes por día (x̄:74,7), el 70\% envía más de 50 mensaje por hora, así mismo, el 76,\% envía más de 5 mensajes por minutos, y escriben rápido más o igual de 30 palabras por minuto. Es importante mencionar que, el 98,3\% de los estudiantes utilizan otros dispositivos, el 71,7\% usa computadora sin mouse, el 65\% los usa durante la noche, y el 60\% los usa menos de 5 horas al día.

Tabla 4. Porcentaje del uso de otros dispositivos.

\begin{tabular}{|l|c|c|}
\hline Uso de otros dispositivos & n & \% \\
\hline Computadora con mouse & 18 & 30 \\
Computadora sin mouse & 43 & 71,7 \\
Tablet & 19 & 31,7 \\
Video juegos & 7 & 11,7 \\
\hline Momento del día & 8 & \\
Por la mañana & 30 & 13.3 \\
Por la tarde & 39 & 50 \\
Por la noche & 14 & 65 \\
Todo el día & & 23,3 \\
\hline Tiempo en horas usando otros dispositivos & 36 & 60 \\
$<5$ horas & 24 & 40 \\
\hline > $=5$ horas & & \\
\hline
\end{tabular}


Los resultados reflejan que el $98,3 \%$ de los estudiantes utilizan otros dispositivos. Datos de la tabla 4, reflejan que el 71,7\% usa computadora sin mouse y el 31,7\% usa tablet; el $65 \%$ lo utilizan durante la noche, y el 60\% los usa menos de cinco horas al día. Datos similares se reportan en el estudio de la universidad de Khon Kaen Tailandia, el 79,63\% usó otros dispositivos, especialmente computadoras y teléfonos inteligentes por la noche, por $3,55 \pm 2,66$ horas al día (Namwongsa et al., 2018).

Síntomas y enfermedades musculoesqueléticas

La prevalencia de síntomas musculoesqueléticos fue del 63,3\%. El síntoma predominante fue el dolor, $41,7 \%$ en cuello, y $35 \%$ y $1,7 \%$ en mano/muñeca derecha e izquierda respectivamente (figura 1).

Namwongsa et al. (2018), presentan que la región más dolorosa después de uso de teléfonos inteligentes durante un periodo de 12 meses, es el cuello (32,5), asociado principalmente a la postura de flexión de cuello $(\mathrm{OR}=2,44$ - IC95\%=1,21-4,90).

Figura 1. Porcentaje de síntomas musculoesqueléticos de cuello y mano/muñeca.

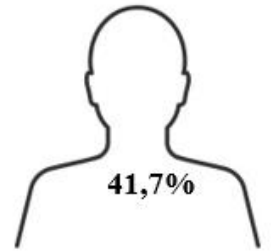

Cuello

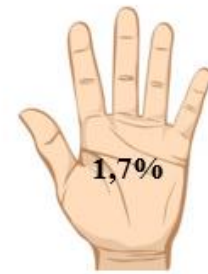

Mano Izquierda

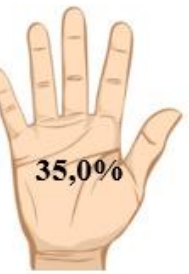

Mano Derecha

Nota. $\mathrm{n}=60$.

Figura 2. Porcentaje de síntomas musculoesqueléticos en cuello y mano/muñeca derecha e izquierda.

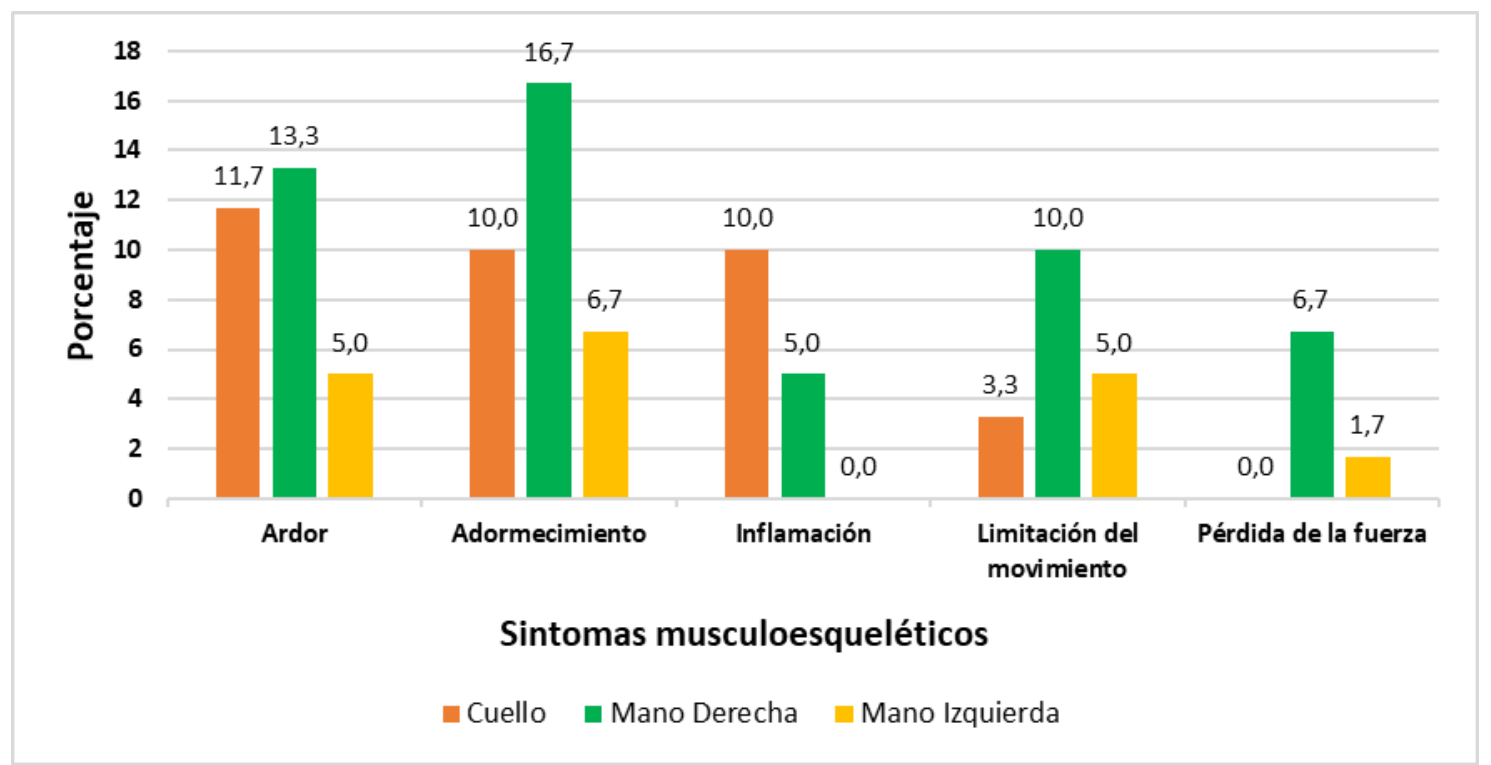

Nota. $\mathrm{n}=60$. Examen físico. 
La figura 2 refleja que, los síntomas predominantes son adormecimiento, ardor y limitación de movimientos, tanto para mano derecha como para cuello.

Figura 3. Frecuencia de las enfermedades musculoesqueléticas en cuello y mano/muñeca derecha.

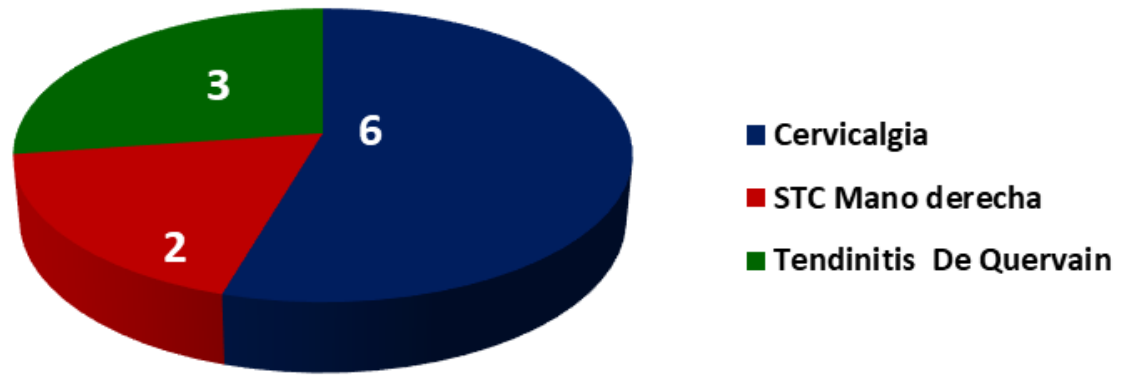

Nota. cuello $(\mathrm{n}=25) ;$ mano/muñeca derecha $(\mathrm{n}=23)$. Examen físico.

En relación al diagnóstico presuntivo de las enfermedades musculoesqueléticas por examen físico, 6 fueron cervicalgia, 2 síndrome del túnel del carpo y 3 tendinitis de Quervain.

\section{Riesgos disergonómicos}

El $100 \%$ de los estudiantes adoptan flexión de cuello mayor a $20^{\circ}$ al utilizar el teléfono celular, el $96,7 \%$ sostiene con ambas manos el teléfono, todos realizan movimientos repetitivos con ambos pulgares para escribir e ingresar a las aplicaciones, y el 48,3\% mantienen postura estática mayor de 8 segundos.

Figura 4. Riesgos disergonómicos de cuello y mano/muñeca al utilizar el teléfono celular.
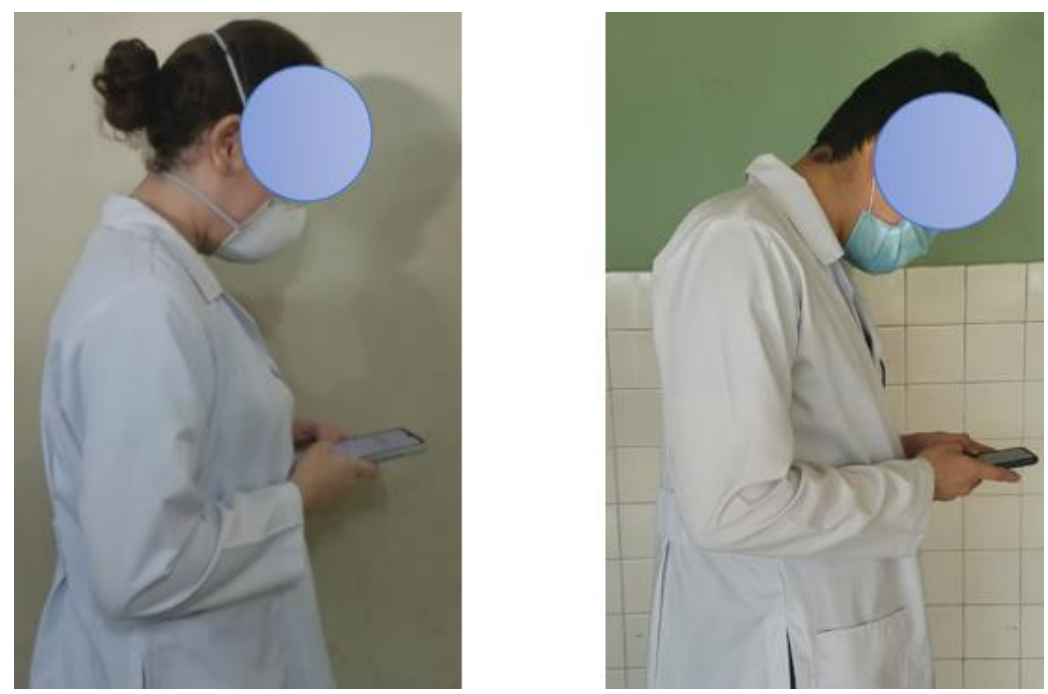

Nota. $\mathrm{n}=60$. Fuente. Videos. 
Aunque los datos difieren, Namwongsa et al. (2018), reportan que al sostener el dispositivo, las posturas que adoptan con mayor frecuencia los usuarios de teléfonos inteligentes son flexión (22,4\%) y supinación de mano/muñeca (21,6\%). Gustafsson et al. (2017), encontraron en un estudio de seguimiento por cinco años, en una población joven entre 20-24 años, asociaciones entre envío de mensaje de textos y los síntomas de cuello y extremidades superiores, manos y dedos.

Según postura forzada de mano/muñeca, el $65 \%$ adopta flexión menor a $15^{\circ}$, el $75 \%$ desviación ulnar de ambas manos y el 28,3\% extensión menor de $15^{\circ}$, al utilizar el teléfono celular.

Figura 5. Postura forzada de mano/muñeca al escribir con el teléfono celular.
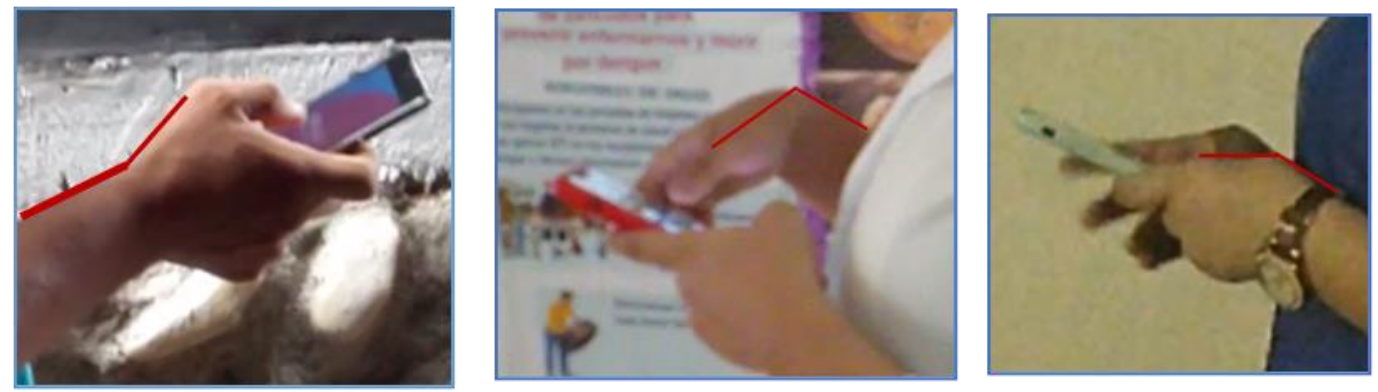

Nota. $\mathrm{n}=60$. Fuente. Videos.

Correa-Botero et al. (2017), mostraron una prevalencia de sintomatología de desórdenes musculoesqueléticos del $45 \%$ (dolor, disconfort, parestesias). El dolor es el síntoma más prevalente con un $24,4 \%$ de los casos, seguido de las parestesias con un $19,4 \%$. Estos síntomas se localizan principalmente en la mano con $29,4 \%$ que corresponde a la inervación del nervio cubital, seguido del $28,8 \%$ inervada por el nervio radial.

Aunque no fue el objetivo del presente estudio, es importante mencionar el estudio realizado por Gustafsson et al. (2018), en el cual se comparó la postura del dedo pulgar al enviar mensajes de textos en teléfono con pantalla táctil y teléfono con teclado, mostrando que el rango de movimiento de flexión era más pequeño, el dedo pulgar estaba menos rotado internamente, y la actividad del pulgar y del antebrazo fue menor; estas diferencias se encontraron en el grupo de manos cortas y se encontraron diferencias en la actividad muscular en el grupo con manos largas. Por tanto, estos hallazgos sugieren que hay diferencias en el riesgo de desarrollar trastornos musculoesqueléticos durante el uso de teléfonos inteligentes con diferentes activaciones de teclas y tamaños de mano.

Los datos presentados en la tabla 5 , muestran que hay 2 veces más probabilidades de dolor musculoesquelético, cuando al utilizar el teléfono celular, existe desviación radial de ambas manos (X2 0,036, IC95\% 1,14-2,56). Por otra parte, se reflejó que existe 7 veces más probabilidad de presentar cervicalgia, al enviar igual o más de 150 SMS por día (X2 0,02, IC95\% 0,99-56,20), sin embargo, es una relación estadísticamente no significativa. 
Tabla 5. Relación entre factores asociados y síntoma de dolor musculoesquelético, en usuarios de teléfono celular.

\begin{tabular}{|c|c|c|c|c|c|c|c|}
\hline \multirow{3}{*}{ Factores asociados } & \multicolumn{4}{|c|}{ Dolor musculoesquelético } & \multirow{3}{*}{$\mathbf{X}^{2}$} & \multirow{3}{*}{$\mathbf{R P}$} & \multirow{3}{*}{ IC (95\%) } \\
\hline & \multicolumn{2}{|c|}{ Sí } & \multicolumn{2}{|c|}{ No } & & & \\
\hline & $\mathbf{F}$ & $\%$ & $\mathbf{F}$ & $\%$ & & & \\
\hline Hombre & 18 & 51,4 & 17 & 48,6 & \multirow{2}{*}{0,6} & \multirow{2}{*}{0,8} & \multirow{2}{*}{$0,54-1,35$} \\
\hline Mujer & 15 & 60 & 10 & 40,0 & & & \\
\hline \multicolumn{8}{|l|}{ Peso (gr) } \\
\hline$>0=170$ & 17 & 56,7 & 13 & 43,3 & \multirow{2}{*}{1,00} & \multirow{2}{*}{1,06} & \multirow{2}{*}{$0,67-1,68$} \\
\hline$<170$ & 16 & 53,3 & 14 & 46,7 & & & \\
\hline \multicolumn{8}{|l|}{ Longitud (mm) } \\
\hline$>0=150$ & 19 & 50 & 13 & 40,6 & \multirow{2}{*}{0,60} & \multirow{2}{*}{0,84} & \multirow{2}{*}{$0,2-1,34$} \\
\hline$<150$ & 14 & 59,4 & 14 & 50,0 & & & \\
\hline \multicolumn{8}{|l|}{ Anchura (mm) } \\
\hline$>0=73$ & 15 & 55,6 & 15 & 45,5 & \multirow{2}{*}{1,00} & \multirow{2}{*}{0,98} & \multirow{2}{*}{$0,62-1,55$} \\
\hline$<73$ & 18 & 54,6 & 12 & 44,4 & & & \\
\hline \multicolumn{8}{|l|}{ Espesor (mm) } \\
\hline$>0=8$ & 12 & 50 & 12 & 50,0 & \multirow{2}{*}{0,6} & \multirow{2}{*}{1,16} & \multirow{2}{*}{$0,71-1,89$} \\
\hline$<8$ & 21 & 58,3 & 15 & 41,7 & & & \\
\hline \multicolumn{8}{|l|}{ Años de uso } \\
\hline$>0=8$ & 17 & 63 & 10 & 37 & \multirow{2}{*}{0,30} & \multirow{2}{*}{0,77} & \\
\hline$<8$ & 16 & 48,5 & 17 & 51,5 & & & $0,48-1,21$ \\
\hline Minutos utilizando el & & & & & & & \\
\hline teléfono al día & 19 & 55,9 & 15 & 44,1 & & & \\
\hline $\begin{array}{l}>0=9 \\
<0\end{array}$ & 14 & 53,8 & 12 & 46,2 & 1,00 & 1,03 & $0,65-1,65$ \\
\hline Tiempo de descanso & & & & & & & \\
\hline$>0=15$ minutos & 11 & 64,7 & 6 & 35,3 & & & \\
\hline$<15$ minutos & 21 & 50 & 21 & 50,0 & 0,39 & 1,29 & $0,81-2,05$ \\
\hline Uso durante la noche & 5 & 45,5 & 6 & 54,5 & 0,52 & 0,79 & $0,3-1,58$ \\
\hline Uso todo el día & 27 & 57,4 & 20 & 42,6 & 0,53 & 1,24 & $0,65-2,35$ \\
\hline $\begin{array}{l}\text { Número de mensajes c } \\
\text { texto en un día }\end{array}$ & & & & & & & \\
\hline$>0=150$ mensajes & 16 & 55,2 & 13 & 44,8 & & & \\
\hline$<150$ mensajes & 17 & 54,8 & 14 & 45,2 & 1,00 & 0,99 & $0,62-1,57$ \\
\hline $\begin{array}{l}\text { Desviación radial de } \\
\text { ambas manos }\end{array}$ & 12 & 80 & 3 & 20 & 0,036 & 2 & $1,14-2,56$ \\
\hline $\begin{array}{l}\text { Desviación ulnar de } \\
\text { ambas manos }\end{array}$ & 21 & 46,7 & 24 & 53,3 & 0,036 & 0,58 & $0,3-0,87$ \\
\hline
\end{tabular}

Nota. $\mathrm{n}=60$.

Lee et al. (2015) encontraron que los participantes mantenían una flexión de la cabeza de $33^{\circ}$ a $45^{\circ}$ al usar teléfono inteligente, siendo significativo para el envío de mensajes de texto y con mayor significación mientras está sentado que de pie, sugiriendo que la mensajería de texto, que es una de las categorías de aplicaciones de teléfonos inteligentes 
más utilizadas, podría ser un factor principal que contribuya a la aparición de dolor de cuello en los usuarios habituales de teléfonos inteligentes. Ayhualem et al. (2021), mostraron que el uso de teléfono por período largo, jugar juegos, no tomar descanso, uso de otros dispositivos electrónicos, y el aumento en el uso de las redes sociales, se asocia con el dolor de cuello entre los usuarios de teléfonos inteligentes.

La principal limitante de esta investigación fue que no se pudo incorporar a toda la población de estudio previamente definida, ya que la recolección de los datos se realizó en el contexto de la pandemia por COVID-19, lo que hizo difícil acceder a todos los estudiantes por la reorganización de las actividades académicas de presencial a virtual. La poca población en estudio, puede explicar la baja prevalencia encontrada de las enfermedades musculoesqueléticas.

\section{Conclusiones}

Este estudio muestra que las características de los teléfonos celulares, como mayor peso, espesor, longitud, el uso de estos, como el envío de más de 150 mensajes al día, y los riesgos disergonómicos, como la desviación radial de la mano y flexión de cuello mayor de 20 grados, son factores de riesgos predisponentes a síntomas musculoesqueléticos por el uso de los teléfonos celulares. Estos hallazgos orientan sobre el origen de posibles enfermedades musculoesqueléticas futuras, tanto en cuello y mano/muñeca, debido al uso continuo de este dispositivo, lo que indica la importancia de continuar investigando esta temática para desarrollar en un futuro programas de promoción de la salud y prevención de la enfermedad musculoesquelética por el uso de teléfonos celulares. 


\section{Referencias}

Al-Hadidi, F., Bsisu, I., AlRyalat, S. A., Al-Zu’bi, B., Bsisu, R., Hamdan, M., Kanaan, T., Yasin, M., \& Samarah, O. (2019). Association between mobile phone use and neck pain in university students: A cross-sectional study using numeric rating scale for evaluation of neck pain. PLoS ONE 14(5), e0217231. https://doi.org/10.1371/journal.pone.0217231

Ayhualem, S., Alamer, A., Dabi, S. D., Bogale, K. G., Abebe, A. B., \& Chala, M. B. (2021). Burden of neck pain and associated factors among smart phone user students in University of Gondar, Ethiopia. PLoS ONE 16(9), e0256794. https://doi.org/10.1371/journal.pone.0256794

Correa-Botero, S., Forero-Corrales, D., García-Hoyos, A., Ruiz-Ceballos, S., \& Vanegas-Toro, C. (2017). Características físicas, conocimiento de efectos y uso de teléfonos inteligentes asociados a síntomas de desórdenes musculo-esqueléticos y Tenosinovitis de Quervain [tesis de pregrado, Universidad CES]. Repositorio Digital Institucional. http://hdl.handle.net/10946/3847

Ericsson Mobility Report (2021, junio). $5 G$ en el camino hacia el mercado masivo. https://www.ericsson.com/mobility-report/

Gustafsson, E., Coenen, P., Campbell, A., \& Straker, L. (2018). Texting with touchscreen and keypad phones - A comparison of thumb kinematics, upper limb muscle activity, exertion, discomfort, and performance. Applied Ergonomics, 70, 232-239. https://doi.org/10.1016/j.apergo.2018.03.003

Gustafsson, E., Johnson, P. W., \& Hagberg, M. (2010). Thumb postures and physical loads during mobile phone use - A comparison of young adults with and without musculoskeletal symptoms. Journal of Electromyography and Kinesiology, 20(1), 127-35. https://doi.org/10.1016/j.jelekin.2008.11.010

Gustafsson, E., Thomée, S., Grimby-Ekman, A., \& Hagberg, M. (2017). Texting on mobile phones and musculoskeletal disorders in young adults: A five-year cohort study. Applied Ergonomics, 58, 208-214. https://doi.org/10.1016/j.apergo.2016.06.012

Korpinen, L. H., \& Pääkkönen, R. J. (2009). Self-report of physical symptoms associated with using mobile phones and other electrical devices. Bioelectromagnetics, 30, 431-437. https://doi.org/10.1002/bem.20500

Lee, S., Kang, H., \& Shin, G. (2015). Head flexion angle while using a smartphone. Ergonomics, 58(2), 220-226. https://doi.org/10.1080/00140139.2014.967311

Namwongsa, S., Puntumetakul, R., Neubert, M. S., \& Boucaut, R. (2018). Factors associated with neck disorders among university student smartphone users. Work, 61(3), 367-378. https://doi.org/10.3233/wor-182819

Oliveira, A., Siquiera, E., Porfirio, S., \& Torres, E. (2016). Associação entre o uso excessivo de smartphones e as lesões de punho e dedos em estudantes de ensino superior da área de saúde. Revista Inspirar. https://repositorio.asces.edu.br/handle/123456789/533

Perera, C. (2012). The evolution of E-Health - Mobile technology and mHealth. Journal of Mobile Technology in Medicine, 1(1), 1-2. http://doi.org/10.7309/jmtm.1 